\title{
Infrequency of Sexually Transmitted Disease Screening Among Sexually Experienced U.S. Female Adolescents
}

\begin{abstract}
CONTEXT: Since 1993, the Centers for Disease Control and Prevention and professional medical organizations have recommended that all sexually experienced female adolescents receive annual screening for Chlamydia trachomatis. Whether adolescents receive this care is largely unknown.
\end{abstract}

\begin{abstract}
METHODS: Reports of receipt of testing or treatment for a sexually transmitted disease (STD) in the past year, as well as sites of care, were obtained from 3,987 sexually experienced females in grades 7-12 who participated in Wave 1 of the National Longitudinal Study of Adolescent Health, conducted in 1995. Logistic regression was used to determine predictors of reporting care.
\end{abstract}

RESULTS: Eighteen percent of all participants reported having received STD services in the past year. Of those who reported having had a routine physical examination in the past year, $22 \%$ reported receipt of STD services. The proportion reporting STD care increased linearly with age from 9\% of 12-13-year-olds to $25 \%$ of those 19 or older. In adjusted analyses, the odds of reporting testing or treatment were elevated among participants who had had a physical examination in the past year (odds ratio, 2.1), those with Medicaid or Medicare insurance (1.9), black women (1.5) and older adolescents (1.2). Adolescents most often reported having received STD care at a community health center (44\%) or a private physician's office (31\%).

CONCLUSIONS: Continued inadequate screening may contribute to persisting high prevalence of chlamydia infection among adolescents. Future research is needed to determine whether the proportions of adolescents receiving recommended STD screening have increased over time.

Perspectives on Sexual and Reproductive Health, 2004, 36(6):233-238
By Lynne C. Fiscus, Carol A. Ford and William C. Miller

Lynne C. Fiscus is a resident, Departments of Pediatrics and Medicine; Carol A. Ford is associate professor of pediatrics and medicine; and William C. Miller is associate professor of epidemiology and medicine-all at the University of North Carolina at Chapel Hill.
The prevalence of chlamydia infection-which is associated with considerable personal, societal and economic costsis high among adolescents in the United States. ${ }^{1}$ By 18 years of age, $4 \%$ of all adolescents are infected with Chlamydia trachomatis, and the prevalence is higher among black, Native American and Hispanic women (13\%, 10\% and 6\%, respectively); the vast majority of these infections are asymptomatic. ${ }^{2}$ Despite the absence of symptoms, infection can ascend to the upper genital tract, causing pelvic inflammatory disease, tubal scarring, increased risk of ectopic pregnancy and infertility. ${ }^{3}$ Asymptomatic infection can also be transmitted to sexual partners, sustaining high prevalence of infection in sexual networks. Adolescents have high rates of repeat infection, and thus are at particularly high risk of medical complications. ${ }^{4}$

Since 1993, the Centers for Disease Control and Prevention (CDC) has recommended that all sexually experienced female adolescents be screened annually for Chlamydia trachomatis. ${ }^{5}$ The American Academy of Pediatrics, the American Medical Association (AMA) and the U.S. Preventive Services Task Force have made similar recommendations, ${ }^{6}$ and annual chlamydia screening among sexually experienced female adolescents is now a quality of care measure in HEDIS (the Health Plan Employer Data and Information Set, a national set of standardized measures of health plan performance). ${ }^{7}$ Despite consensus in professional recommenda- tions, regional research suggests that many sexually experienced female adolescents are not receiving annual screening for chlamydia infection. ${ }^{8}$ To our knowledge, there are no published national estimates of the proportion of sexually experienced female adolescents who report receiving annual screening for sexually transmitted diseases (STDs).

Wave 1 of the National Longitudinal Study of Adolescent Health (Add Health), conducted in 1995, provides the opportunity to describe STD testing patterns among a large, nationally representative sample of adolescents approximately 2-3 years after the CDC and AMA began recommending universal annual screening for chlamydia infection among sexually experienced female adolescents. It also provides the opportunity to describe baseline receipt of STD testing services, which can be compared with later testing patterns. Given persisting high rates of curable STDs among adolescents, identifying trends in and patterns of STD testing within this age-group is important.

In this study, we describe the proportion of sexually experienced female adolescents in Wave 1 of Add Health who reported having received testing or treatment for an STD in the past year, and how reported receipt of STD-related health care varied by age, race and ethnicity, insurance status and whether participants had had a recent routine physical examination. We also examine what types of clinical sites adolescents reported using for STD care. 


\begin{tabular}{|c|c|c|c|}
\hline Characteristic & $\begin{array}{l}\text { All } \\
\text { respondents } \\
(\mathrm{N}=3,987)^{*}\end{array}$ & $\begin{array}{l}\text { Tested or } \\
\text { treated } t\end{array}$ & Odds ratio \\
\hline Total & 100.0 & 18.2 & na \\
\hline \multicolumn{4}{|l|}{ Age } \\
\hline $12-13$ & 3.2 & 9.1 & $0.26(0.07-0.90)$ \\
\hline 14-15 & 21.4 & 13.9 & $0.40(0.19-0.84)$ \\
\hline 16 & 22.4 & 18.6 & $0.61(0.34-1.12)$ \\
\hline 17 & 27.5 & 19.1 & $0.54(0.30-0.98)$ \\
\hline 18 & 22.8 & 20.8 & $0.66(0.36-1.20)$ \\
\hline$\geq 19$ & 2.8 & 25.0 & ref \\
\hline \multicolumn{4}{|l|}{ Race } \\
\hline Black & 28.8 & 22.2 & ref \\
\hline White & 50.5 & 15.8 & $0.60(0.45-0.82)$ \\
\hline Hispanic & 6.5 & 16.2 & $0.68(0.38-1.21)$ \\
\hline Native American & 1.5 & 32.0 & $1.92(0.93-3.95)$ \\
\hline Asian & 4.6 & 15.8 & $0.95(0.54-1.69)$ \\
\hline Other & 8.1 & 19.1 & $0.57(0.35-0.93)$ \\
\hline \multicolumn{4}{|l|}{ Insurance } \\
\hline Medicaid/Medicare & 11.8 & 28.6 & ref \\
\hline Individual/group & 34.9 & 14.1 & $0.44(0.32-0.60)$ \\
\hline Prepaid HMO & 18.1 & 18.5 & $0.52(0.33-0.80)$ \\
\hline Other/don't know & 4.5 & 16.6 & $0.70(0.40-1.21)$ \\
\hline None & 11.7 & 16.0 & $0.54(0.36-0.81)$ \\
\hline Missing & 19.0 & 21.0 & $0.69(0.47-1.02)$ \\
\hline \multicolumn{4}{|c|}{ Physical exam in past year } \\
\hline Yes & 65.9 & 21.9 & $2.20(1.73-2.81)$ \\
\hline No & 34.1 & 10.8 & ref \\
\hline
\end{tabular}

*Unweighted data. PPercentages account for oversampling in the data set to obtain a nationally representative sample. Note: ref=reference group.

\section{METHODS}

\section{Study Design}

Add Health's sampling design and procedures have been described in detail elsewhere. ${ }^{9}$ In summary, respondents were a systematically drawn random sample of adolescents in grades 7-12 from 132 schools in the United States. The sample for this study is restricted to the 3,988 female Wave 1 participants who responded yes to the question "Have you ever had sexual intercourse? When we say sexual intercourse we mean when a male inserts his penis into a female's vagina."

Informed written parental consent and minor assent were required for participation. Adolescents completed a 90minute in-home interview on a wide range of topics that influence adolescent health and well-being. Interviews were conducted in as private an area as possible and were performed from April to December 1995. Sensitive portions of the interview, including questions concerning sexual activity, were performed through audio computer-assisted self-interview. This technique promotes more complete reporting of sensitive topics than face-to-face interviews. ${ }^{10}$ Whenever possible, parents were also interviewed. All procedures for Add Health were approved by the University of North Carolina Institutional Review Board.

\section{Data and Analysis}

Measures of receipt of specific STD services were not available. Therefore, the outcome variable, receipt of STD care, was measured by responses to the question "In the past year, have you received testing or treatment for a sexually transmitted disease or AIDS?"

Most predictor variables were derived from data provided by adolescent participants. Age was calculated from date of birth. Race and ethnicity were measured by self-report. All participants who responded yes to the question "Are you of Hispanic or Latino origin?" were categorized as Hispanic. Other participants were categorized as black or African American, white, Native American or American Indian, Asian or Pacific Islander, or other. Participants who reported more than one racial category were asked "Which one category best describes your racial background?" and were categorized on the basis of their response. Receipt of a routine physical in the year prior to the interview was measured by the question "In the past year have you had a routine physical examination?" Respondents who reported they had received STD testing or treatment in the past year were asked "Where did you receive this testing or treatment?" Response categories were private doctor's office, community health center, school, hospital or some other place.

Data for one measure, participants' insurance status, were taken from the parents survey. Response options were Medicaid, Medicare, individual or group, prepaid health maintenance organization (HMO), other, none and don't know.

Frequencies to describe our sample were calculated from unweighted data. Crude prevalence of self-reported testing for STDs and prevalence odds ratios with 95\% confidence intervals were determined using STATA 7.0, employing survey commands and weighted data for all sexually active female adolescents who answered the question on receipt of services for an STD in the past year. Use of sample weights and adjustments for the clustered sampling design provide estimates for a nationally representative sample.

We used simple logistic regression models to calculate prevalence odds ratios with $95 \%$ confidence intervals for individual variables. Variables that were associated with reporting receipt of STD services at $p<0.2$ in these models were included in a multiple logistic regression model. We used a backward elimination procedure to identify independent predictors of reporting receipt of STD care in the past year. Variables were removed one at a time from the model until only factors statistically significantly associated with the outcome at $\mathrm{p}<.05$ remained. The proportions of sexually experienced female adolescents who said they had received care at particular types of clinic sites were also determined.

\section{RESULTS \\ Participants}

All but one of the 3,988 female adolescents in this sample indicated whether they had received care for an STD in the past year. Participants' mean age was 16.4 years (standard error, 0.07; range, 12-21). Fifty-one percent of participants 
were white, 29\% were black and 7\% were Hispanic; the remainder were Asian, Native American or members of other racial or ethnic groups (Table 1). Some 35\% of participants were covered by individual or group insurance, $18 \%$ by an $\mathrm{HMO}$ and $12 \%$ by Medicaid or Medicare; $12 \%$ were uninsured, and data for this variable were not reported for 19\%. Two-thirds of respondents said they had had a routine physical examination in the past year.

\section{STD Care in Past Year}

Overall, 18\% of participants reported having been tested or treated for STDs in the past year. The proportion increased linearly as females progressed through adolescence. The difference in prevalence of self-reported testing or treatment between 12-13-year-olds and respondents aged 19 or older was 16 percentage points ( $9 \%$ vs. $25 \%$ ); results of the bivariate analysis indicate that the youngest adolescents were significantly less likely than the oldest respondents to report testing or treatment (odds ratio, 0.3 ).

One-third (32\%) of Native American adolescents reported that they had been tested or treated for STDs in the past year, as did 22\% of blacks and 16-19\% of adolescents in the remaining racial groups. Compared with black adolescents, white adolescents and those of "other" races were less likely to report having been tested or treated for STDs (odds ratio, 0.6 for each). In another bivariate model (not shown), the odds of a black female adolescent's reporting having been tested or treated for an STD were 1.6 times the odds of all other adolescents (95\% confidence interval, 1.2-2.1).

Compared with participants who had Medicaid or Medicare coverage, those with individual or group insurance coverage and those with prepaid HMO insurance were significantly less likely to report having received STD care in the past year (odds ratios, 0.4 and 0.5, respectively). Uninsured participants also had reduced odds of reporting testing or treatment (0.5). The odds that a sexually experienced female with Medicaid or Medicare reported having been tested or treated for an STD were 2.1 times the odds of all other groups (95\% confidence interval, 1.6-2.8-not shown).

Of the $66 \%$ of sexually experienced female adolescents who reported having had a routine physical examination in the past year, only $22 \%$ said they had received STD testing or treatment. Although low, this rate is significantly higher than that among participants who did not report having undergone a routine physical examination in the past year (odds ratio, 2.2).

In multivariate analyses, four variables were associated with the odds of reporting receipt of STD services (Table 2). The strongest predictor was reported receipt of a routine physical examination in the past year: Adolescents who said they had undergone a routine physical examination had twice the odds of reporting testing when compared with others (odds ratio, 2.1). The odds were nearly doubled for adolescents with Medicaid or Medicare (1.9), and were about half again as high among blacks as among others (1.5). Also, the older the adolescent, the greater the odds of reporting testing (1.2).
TABLE 2. Odds ratios (and 95\% confidence intervals) from logistic regression analysis of characteristics associated with sexually experienced female adolescents' reporting receipt of STD testing or treatment within the past year

Characteristic

Odds ratio

Increasing age

$1.20(1.07-1.35)$

Black

$1.49(1.05-2.11)$

Medicaid/Medicare

$1.94(1.45-2.58)$

Physical exam in past year

$2.08(1.54-2.82)$

Note: Age is measured as an ordered categorical variable (see Table 1); all other characteristics are measured dichotomously.

\section{Reported Sites of STD Care}

Of the 740 participants who reported receipt of STD care, the largest proportion (44\%) said they had gotten that care at a community health center; a private physician's office was the next most commonly reported source of care (31\%). Hospitals and, especially, school-based clinics and other sites were reported far less frequently (17\%, 3\% and 4\%, respectively).

\section{DISCUSSION}

This first national estimate of self-reported STD care among sexually experienced U.S. female adolescents suggests that in the mid-1990s, only one in five were receiving recommended screening, even if they were receiving routine health care. This surprisingly low estimate may lead to questions about measurement error. Perhaps many adolescents were not aware that they were screened for STDs, and our results, based on self-reported data, may substantially underestimate actual STD screening. The risk of this potential bias is minimized because data collection took place prior to the use of urine testing for STDs in clinical settings, when chlamydia testing required a pelvic examination. Pelvic examinations are a highly salient health care event. The test-retest reliability among high school students asked whether they received chlamydia testing in the past 12 months and whether they underwent a pelvic examination at their last checkup has been relatively high (kappas, 0.8 and 0.9 , respectively). ${ }^{11}$ In one study, the sensitivity and specificity of questions about Chlamydia trachomatis testing 4-6 months after a clinic visit among adolescents aged 14-21 were 100\% and 93\%, respectively, suggesting that the validity of self-report questions is excellent. ${ }^{12}$ Although this information is reassuring, it remains possible that respondents either received pelvic examinations and were unaware that they were tested for STDs or assumed that they were tested for STDs when, in fact, they were not. Both of these situations would lead to measurement error-but in opposite directions.

Nonetheless, because our national prevalence estimate of self-reported rates of STD care is extremely low, we are concerned that our results suggest widespread deficiencies in the quality of health care received by sexually experienced female adolescents. Our estimate is much lower than the annual prevalence of STD testing among sexually experienced female adolescents (59\%) reported in a regional study conducted at approximately the same time, 
but that telephone survey focused on an identified highrisk group of adolescents in neighborhoods with known high STD rates. ${ }^{13}$ Our estimate falls within the wide range of chlamydia screening rates (2-42\%) found among 15-25year-old women in four geographically dispersed managed care plans in $1997 .{ }^{14}$

Furthermore, our results suggest that this deficiency in quality of health care occurs even among adolescents who are receiving routine health care. Two-thirds of our sample reported having had a routine physical examination in the previous year. Similarly, $64 \%$ of sexually experienced female participants in the Youth Risk Behavior Survey reported having had a preventive office visit in the past year. ${ }^{15}$ We found that having received a routine physical examination in the past year was positively associated with reporting testing or treatment for STDs. However, only $22 \%$ of all adolescents who reported having had a routine physical examination said they had gotten STD care. This observation is striking because it suggests that barriers to accessing the health care system are not a major determinant of who receives STD care for the majority of U.S. adolescents.

Overall low levels of STD screening among sexually experienced adolescents likely have multiple explanations. First, it takes time for any new recommendation to be implemented. Our study occurred 2-3 years after recommendations for universal screening among sexually experienced female adolescents were published, and diffusion of the recommendation into clinical care may have taken much longer. Population-based studies using more recent data are needed to determine whether STD screening rates among sexually experienced female adolescents have improved.

Second, practitioners' adherence to screening recommendations varies widely. ${ }^{16}$ Female physicians and physicians who have more recently graduated from medical school are more likely than males and less recent graduates to screen adolescents for STDs. ${ }^{17}$ Obstetrician-gynecologists are more likely to offer STD counseling than are pediatricians or general practitioners, although results are conflicting in regard to screening practices of obstetriciangynecologists and pediatricians. ${ }^{18}$

Third, adherence to screening recommendations varies by site of care. Public health clinics providing care to adolescents see patients of lower socioeconomic status and with higher rates of risky behavior than private clinic settings, 19 and providers in public settings may have a heightened awareness of the need for STD screening. Practicing within an $\mathrm{HMO}$ and having a higher proportion of patients belonging to an $\mathrm{HMO}$ are also associated with increased STD testing. ${ }^{20}$ Interestingly, although most adolescents receive their regular health care at private offices, ${ }^{21}$ the largest proportion of Add Health participants reported going to community health centers for STD care. Privacy is a significant factor for teenagers in determining whether and where they go for STD services. ${ }^{22}$ These concerns may lead adolescents to seek STD care at sites other than their primary care physicians' offices.

Finally, providers' bias concerning which adolescents are likely to be at risk for STDs probably leads to differences in who is tested. Judgments may be related to patients' age and race. We found that reported testing or treatment for STDs among sexually experienced female adolescents increases incrementally as teenagers progress through adolescence. This pattern may reflect increased inclusion of sexual histories in routine care as female adolescents get older and make a transition away from seeing a pediatrician and toward care that includes visits to an obstetriciangynecologist. In a survey of physicians, Ziv found that by the time female adolescents were 18-21 years old, 37\% of their physician visits were to obstetricians and gynecologists; in the same study, these specialists were more likely to offer counseling concerning STDs than were pediatricians or general practitioners. ${ }^{23}$ Similarly, racial bias may play a role in differential testing patterns for STDs among adolescents. Blacks and Native Americans in Add Health reported the highest prevalence of STD care in the past year.

The relationship between insurance status of adolescent patients and STD testing patterns is striking. We found that participants with individual or group or prepaid HMO coverage were much less likely to report having received STD care than were those with Medicaid or Medicare. A provider survey has found that private for-profit sites are less likely to routinely screen for Chlamydia trachomatis than are other types of sites (private nonprofit, community health center, health department or university health center). ${ }^{24}$ These disparities may reflect biases in clinicians' perceptions of STD risk among patients seen in for-profit versus not-for-profit sites and by insurance status, or different standards of care for adolescents (with privately insured adolescents and those attending for-profit clinics receiving lower-quality care than comparison groups). Financial risk may disproportionately influence testing behaviors in for-profit clinical sites. Finally, it may be more difficult to provide confidential STD testing in for-profit sites because of paper trails related to billing and explanations of benefits. ${ }^{25}$

\section{Limitations}

Limitations of the study include the use of an outcome measure that addressed STD testing and treatment simultaneously. We assume that treatment implies that testing was performed. Persons receiving treatment may be more likely to have been symptomatic, rather than tested for asymptomatic disease. Consequently, another possible contributor to the increased testing across age-groups is an associated increase in incident infections resulting in careseeking and treatment. The characteristics of persons seeking care for symptoms and receiving treatment may be considerably different from those of individuals undergoing testing for screening purposes.

Furthermore, the wording of the Add Health measure for sexual experience excludes participants who have engaged exclusively in homosexual intercourse, and the results of our study may not apply to this important group. Finally, the original Add Health sample was drawn from school enrollment rosters. Consequently, persons who had 
dropped out of school prior to Wave 1 were not included. These individuals may be more likely to engage in sexual intercourse, be at higher risk for STDs, and have different use of STD testing services. However, previous research suggests that potential bias because of missing school dropouts in Add Health is small. ${ }^{26}$

\section{CONCLUSIONS}

The vast majority of sexually experienced U.S. female adolescents did not report receiving testing for STDs in 1995, in accordance with national guidelines. Barriers to accessing health care do not fully explain this lack of testing, as only $22 \%$ of those who reported receiving a routine physical reported being tested or treated for STDs. Future research is urgently needed to determine whether STD screening practices in adolescent populations have improved over time, to better understand variation in compliance with current STD screening recommendations, and to more clearly define the relationship between clinic-based screening and community rates of STDs. Additionally, more work is needed to ensure that adolescents understand the benefits of STD screening and to encourage their active role in being screened.

\section{REFERENCES}

1. Institute of Medicine, The Hidden Epidemic: Confronting Sexually Transmitted Diseases, Washington, DC: National Academy Press, 1997; Weinstock H, Berman S and Cates W, Jr., Sexually transmitted infections among American youth: incidence and prevalence estimates, Perspectives on Sexual and Reproductive Health, 2004, 36(1):6-10; Chesson HW et al., The estimated direct medical cost of sexually transmitted diseases among American youth, 2000, Perspectives on Sexual and Reproductive Health, 2004, 36(1):11-19; and American Academy of Pediatrics (AAP), Red Book: 2003 Report of the Committee on Infectious Diseases, 26th ed., Elk Grove, IL: AAP, 2003, pp. 238-243.

2. Miller $\mathrm{W}$ et al., Prevalence of chlamydial and gonococcal infections among young adults in the United States, Journal of the American Medical Association, 2004, 291(18):2229-2236.

3. AAP, 2003, op. cit. (see reference 1); Hillis SD et al., Recurrent chlamydial infections increase the risk of hospitalization for ectopic pregnancy and pelvic inflammatory disease, American Journal of Obstetrics and Gynecology, 1997, 178(1):104-108.

4. Whittington WL et al., Determinants of persistent and recurrent Chlamydia trachomatis infection in young women: results of a multicenter cohort study, Sexually Transmitted Diseases, 2001, 28(2):117-123; Rietmeijer CA et al., Incidence and repeat infection rates of Chlamydia trachomatis among male and female patients in an STD clinic: implications for screening and rescreening, Sexually Transmitted Diseases, 2002, 29(2):65-72; Richey CM, Macaluso M and Hook EW, Determinants of re-infection with Chlamydia trachomatis, Sexually Transmitted Diseases, 1999, 26(1):4-11; Hillis SD et al., Risk factors for recurrent Chlamydia trachomatis infection in women, American Journal of Obstetrics and Gynecology, 1994, 170(3):801-806; and Xu F et al., Repeat Chlamydia trachomatis infection in women: analysis through a surveillance case registry in Washington State, 1993-1998, American Journal of Epidemiology, 2000, 152(12):1164-1170.

5. Centers for Disease Control and Prevention (CDC), Sexually Transmitted Disease Surveillance, 2002, Atlanta: CDC, 2003; CDC, Recommendations for the prevention and management of Chlamydia trachomatis infections, 1993, Morbidity and Mortality Weekly Report, 1993, 42(RR-12):7-8; and CDC, 1998 guidelines for treatment of sexually transmitted disease, Morbidity and Mortality Weekly Report, 1998, 47(RR1):1-118.

6. Elster AB, Comparison of recommendations for adolescent clinical preventive services developed by national organizations, Archives of
Pediatrics and Adolescent Medicine, 1998, 152(2):193-198; Committee on Psychosocial Aspects of Child and Family Health, Guidelines for Health Supervision III, Elk Grove Village, IL: AAP, 1996; Elster AB and Kuznets NJ, AMA Guidelines for Adolescent Preventive Services (GAPS): Recommendations and Rationale, Baltimore: Williams \& Wilkins, 1994; and U.S. Preventive Services Task Force, Screening for chlamydial infection: recommendations and rationale, American Journal of Preventive Medicine, 2001, 20(3S):90-94.

7. National Committee for Quality Assurance, HEDIS 2000, <http:// www.ncqa.org/Programs/HEDIS/h00meas.htm>, accessed Nov. 3, 2004.

8. Ellen JM, Lane MA and McCright J, Are adolescents being screened for sexually transmitted diseases? a study of low income African American adolescents in San Francisco, Sexually Transmitted Infections, 2000, 76(2):94-97; and Mangione-Smith R, McGlynn EA and Hiatt L, Screening for chlamydia in adolescents and young women, Archives of Pediatrics and Adolescent Medicine, 2000, 154(11):1109-1113.

9. Bearman PS, Jones J and Udry JR, The National Longitudinal Study on Adolescent Health: research design, Chapel Hill, NC: Carolina Population Center, 1997, <http://www.cpc.unc.edu/projects/addhealth/design>, accessed Nov. 3, 2004; and Resnick MD et al., Protecting adolescents from harm: findings from the National Longitudinal Study on Adolescent Health 1997, Journal of the Americal Medical Association, 278(10):823-832.

10. Des Jarlais DC et al., Audio-computer interviewing to measure risk behaviour for HIV among injecting drug users: a quasi-randomized trial, Lancet, 1999, 353(9165):1657-1661.

11. SantelliJ et al., Reliability in adolescent reporting of clinician counseling, health care use, and health behaviors, Medical Care, 2002, 40(1): 26-37.

12. Klein JD et al., Developing quality measures for adolescent care: validity of adolescents' self-reported receipt of preventive health services, Health Services Research, 1999, 34(1):391-404.

13. Ellen JM, Lane MA and McCright J, 2000, op. cit. (see reference 8). 14. Mangione-Smith R, McGlynn EA and Hiatt L, 2000, op. cit. (see reference 8).

15. Burstein GR et al., Missed opportunities for sexually transmitted disease, human immunodeficiency virus, and pregnancy prevention services during adolescent health supervision visits, Pediatrics, 2003, 111(5):996-1001

16. Cook RL et al., Barriers to screening sexually active adolescent women for chlamydia: a survey of primary care physicians, Journal of Adolescent Health, 2001, 28(3):204-210; and Torkko KC et al., Testing for chlamydia and sexual history taking in adolescent females: results from a statewide survey of Colorado primary care providers, Pediatrics, 2000, 106(3), <http://www.pediatrics.org/cgi/content/full/106/103/ e132>, accessed Nov. 3, 2004.

17. Cook RL et al., 2001, op. cit. (see reference 16); Torkko KC et al., 2000, op. cit. (see reference 16); and Millstein SG, Igra V and Gans J, Delivery of STD/HIV preventive services to adolescents by primary care physicians, Journal of Adolescent Health, 1996, 19(4):249-257.

18. Cook RL et al., 2001, op. cit. (see reference 16); Torkko KC et al., 2000, op. cit. (see reference 16); Ziv A, Boulet JR and Slap GB, Utilization of physician offices by adolescents in the United States, Pediatrics, 1999 , 104(1):35-42; and Leone PA et al., Chlamydia screening practices of primary-care providers-Wake County, North Carolina, 1996, 1997, 46(35):819-822.

19. Fisher M, Marks A and Trieller K, Meeting the health care needs of suburban youth: review of a clinical service, Pediatrics, 1998, 81(1):8-13.

20. Cook RL et al., 2001, op. cit. (see reference 16); and Ziv A, Boulet JR and Slap GB, 1999, op. cit. (see reference 18).

21. Klein JD et al., Access to medical care for adolescents: results from the 1997 Commonwealth Fund survey of the health of adolescent girls, Journal of Adolescent Health, 1999, 25(2):120-130.

22. Sugerman $S$ et al., Family planning clinic clients: their usual health care providers, insurance status, and implications for managed care, Journal of Adolescent Health, 2000, 27(1):25-33; and Ford C, Best D and Miller W, Confidentiality and adolescents' willingness to consent to STD testing, Archives of Pediatrics and Adolescent Medicine, 2001, 155(9):1072-1073. 
23. Ziv A, Boulet JR and Slap GB, 1999, op. cit. (see reference 18).

24. Leone PA et al., 1996, op. cit. (see reference 18).

25. English A and Ford CA, The HIPAA privacy rule and adolescents: legal questions and clinical challenges, Perspectives on Sexual and Reproductive Health, 2004, 36(2):80-86.

26. Udry JR and Chantala K, Missing school dropouts in surveys does not bias risk estimates, Social Science Research, 2003, 32(2):294-311.

\section{Acknowledgments}

Support for this project was provided in part by the University of North Carolina STD Clinical Research Center (National Institute of Allergy and Infectious Diseases grant U0131496), the National
Institutes of Health (grant HD38210) and The Robert Wood Johnson Foundation Generalist Physician Faculty Scholar Award Program. This research uses data from Add Health, a program project designed by J. Richard Udry, Peter S. Bearman and Kathleen Mullan Harris, and funded by a grant P01-HD31921 from the National Institute of Child Health and Human Development, with cooperative funding from 17 other agencies. Special acknowledgment is due to Ronald R. Rindfuss and Barbara Entwisle for assistance in the original design. Persons interested in obtaining data files from Add Health should contact Add Health, Carolina Population Center, 123 W. Franklin Street, Chapel Hill, NC 27516-2524 (www.cpc.unc.edu) addhealth/contract.html).

Author contact: caf@med.unc.edu 